\title{
ENTROPY DEGENERATION OF CONVEX PROJECTIVE SURFACES
}

\author{
XIN NIE
}

\begin{abstract}
We show that the volume entropy of the Hilbert metric on a closed convex projective surface tends to zero as the corresponding Pick differential tends to infinity. The proof is based on the fact, due to Benoist and Hulin, that the Hilbert metric and the Blaschke metric are comparable.
\end{abstract}

\section{Introduction AND STATEMENT OF RESUlts}

Let $\Sigma$ be a closed oriented surface of genus at least 2. A strictly convex real projective structure (referred to simply as "convex projective structure" in the sequel) is by definition a $\left(\operatorname{PSL}(3, \mathbb{R}), \mathbb{R P}^{2}\right)$ geometric structure whose developing map is a homeomorphism from the universal cover $\widetilde{\Sigma}$ to a bounded convex open set in an affine chart $\mathbb{R}^{2} \subset \mathbb{R P}^{2}$.

It is a theorem of J. Loftin [Lof01] and F. Labourie [Lab07] that the moduli space $\mathcal{P}(\Sigma)$ of convex projective structures on $\Sigma$ naturally identifies with the moduli space $\mathcal{C}(\Sigma)$ of pairs $(\boldsymbol{J}, \boldsymbol{b})$, where $\boldsymbol{J}$ is a conformal structure on $\Sigma$ and $\boldsymbol{b}$ is a holomorphic cubic differential on the Riemann surface $(\Sigma, \boldsymbol{J})$. The space $\mathcal{C}(\Sigma)$ is a holomorphic vector bundle of rank $5(g-1)$ over the Teichmüller space $\mathcal{T}(\Sigma)$, the fiber over $(\boldsymbol{J}, \boldsymbol{b})$ being the space $H^{0}\left((\Sigma, \boldsymbol{J}), K^{3}\right)$ of holomorphic cubic differentials on $(\Sigma, \boldsymbol{J})$. The natural inclusion $\mathcal{T}(\Sigma) \subset \mathcal{P}(\Sigma) \cong \mathcal{C}(\Sigma)$ identifies $\mathcal{T}(\Sigma)$ as the zero section of the vector bundle.

Taking the volume entropy of the Hilbert metric (see Section 2 below for the definitions) for each element of $\mathcal{P}(\Sigma)$ yields a function Ent : $\mathcal{P}(\Sigma) \rightarrow \mathbb{R}_{+}$.

The Hilbert metric for a point in the Teichmüller space $\mathcal{T}(\Sigma) \subset \mathcal{P}(\Sigma)$ is just the hyperbolic metric representing that point. Its entropy is just 1 . On the other hand, M. Crampon proved in Cra09 that Ent is strictly less than 1 outside $\mathcal{T}(\Sigma)$. Motivated by a question of Crampon, we constructed in [Nie] certain paths in $\mathcal{P}(\Sigma)$ along which Ent tends to 0 (the construction works for dimensions 3 and 4 as well, though here we only look at dimension 2). Using different techniques, T. Zhang constructed in Zha13 some submanifolds of $\mathcal{P}(\Sigma)$ with the same property. In view of these constructions, one might naturally guess that Ent tends to 0 along any sequence going away from $\mathcal{T}(\Sigma)$. The purpose of this note is to prove such a statement. Namely,

Received by the editors May 28, 2015 and, in revised form, November 11, 2015.

2010 Mathematics Subject Classification. Primary 51H20, 53C23, 37A35.

The research leading to these results has received funding from the European Research Council under the European Community's seventh Framework Programme (FP7/2007-2013)/ERC grant agreement no. FP7-246918. 
Theorem. Fix a conformal structure $\boldsymbol{J}$ on $\Sigma$. Let $\left(\boldsymbol{b}_{n}\right)$ be a sequence in the space of cubic differentials $V:=H^{0}\left((\Sigma, \boldsymbol{J}), K^{3}\right)$ and let $\delta_{n}$ be the volume entropy of the Hilbert metric of the convex projective structure corresponding to $\left(\boldsymbol{J}, \boldsymbol{b}_{n}\right)$. Then $\delta_{n}$ tends to 0 as $n \rightarrow+\infty$ if and only if $\boldsymbol{b}_{n}$ tends to infinity in $V$.

The "only if" part is an immediate consequence of the continuity of the map Ent $: \mathcal{P}(\Sigma) \rightarrow \mathbb{R}_{+}$and the continuity of the Labourie-Loftin bijection $\mathcal{P}(\Sigma) \cong \mathcal{C}(\Sigma)$. However, to the knowledge of the author, a proof of the latter continuity does not exist in the literature, so we will give an alternative proof of the above theorem without using continuity.

The theorem is a simple manifestation of the general philosophy that a degenerating convex projective surface looks bigger and bigger and more and more flat. See [Lof07, Par12] for more circumstantial statements confirming this philosophy.

\section{The PROOF AND SPECUlations}

First, we briefly review the backgrounds.

Let $\stackrel{\Sigma}{\Sigma}$ be the universal cover of $\Sigma$ and let $\Gamma=\pi_{1}(\Sigma)$ be the fundamental group. Let $g$ be either a Riemannian metric, a Finsler metric or a flat metric with conic singularities on $\Sigma$. Fix a base point $x_{0} \in \widetilde{\Sigma}$. The volume entropy of $g$ can be defined as

$$
\operatorname{Ent}(g)=\limsup _{R \rightarrow+\infty} \frac{1}{R} \log \#\left(B_{g}\left(x_{0}, R\right) \cap \Gamma . x_{0}\right) \in \mathbb{R}_{\geq 0} \cup\{+\infty\},
$$

where the symbol "\#" means taking the cardinal of a set, while $\Gamma . x_{0}$ is the $\Gamma$-orbit of $x_{0}$ and $B_{g}\left(x_{0}, R\right) \subset \widetilde{\Sigma}$ is the ball of radius $R$ centered at $x_{0}$ with respect to the distance on $\widetilde{\Sigma}$ induced by the lift of $g$.

We will make use of the following properties of the entropy:

- The scaling property: $\operatorname{Ent}(t g)=t^{-1} \operatorname{Ent}(g)$ for any $t>0$.

- Given metrics $g_{1}$ and $g_{2}$, if their induced distances $d_{1}$ and $d_{2}$ on $\widetilde{\Sigma}$ are quasi-isometric in the sense that

$$
a^{-1} d_{1}(\cdot, \cdot)-b \leq d_{2}(\cdot, \cdot) \leq a d_{1}(\cdot, \cdot)+b,
$$

for some constants $a>1$ and $b>0$, then

$$
a^{-1} \operatorname{Ent}\left(g_{1}\right) \leq \operatorname{Ent}\left(g_{2}\right) \leq a \operatorname{Ent}\left(g_{1}\right) .
$$

- A theorem of Katok Kat88: for any Riemannian metric $g$ on $\Sigma$, the normalized entropy $\operatorname{Ent}(g)^{2} \cdot \operatorname{Area}(g)$ (which is invariant under scaling) satisfies

$$
\operatorname{Ent}(g)^{2} \cdot \operatorname{Area}(g) \geq 2 \pi|\chi(\Sigma)|=\operatorname{Ent}\left(g_{\text {hyp }}\right)^{2} \cdot \operatorname{Area}\left(g_{\text {hyp }}\right)
$$

where $g_{\text {hyp }}$ is any hyperbolic metric on $\Sigma$ and $\chi(\Sigma)$ is the Euler characteristic of $\Sigma$. Furthermore, equality occurs if and only if $g$ is hyperbolic.

An important consequence of the second property is that

$$
0<\operatorname{Ent}(g)<+\infty \text {. }
$$

This follows from the Švarc-Milnor Lemma, which implies that the distance on $\widetilde{\Sigma}$ induced by a geodesic metric is isometric to the hyperbolic plane.

A convex projective structure on $\Sigma$ gives rise to the following objects on $\Sigma$ (see [Lof01, Lab07, BH13, Nie15] for details):

- a Finsler metric $g_{\mathrm{H}}$, called the Hilbert metric;

- a Riemannian metric $g_{\mathrm{B}}$, called the Blaschke metric; 
- a holomorphic cubic differential $\boldsymbol{b}$ with respect to the conformal structure underlying $g_{\mathrm{B}}$, called the Pick differential.

Moreover, $g_{\mathrm{B}}$ and $\boldsymbol{b}$ satisfy Wang's equation

$$
\kappa_{g_{\mathrm{B}}}=-1+2\|\boldsymbol{b}\|_{g_{\mathrm{B}}}^{2} .
$$

Here $\kappa_{g_{\mathrm{B}}}$ is the curvature of $g_{\mathrm{B}}$ and $\|\boldsymbol{b}\|_{g_{\mathrm{B}}}^{2}$ is the pointwise norm of $\boldsymbol{b}$ with respect to $g_{\mathrm{B}}$, namely, if $g_{\mathrm{B}}=h(z)|\mathrm{d} z|^{2}$ and $\boldsymbol{b}=b(z) \mathrm{d} z^{3}$ in a local coordinate $z$, then

$$
\|\boldsymbol{b}\|_{g_{\mathrm{B}}}^{2}(z):=\frac{|b(z)|^{2}}{h(z)^{3}} .
$$

The Labourie-Loftin theorem says that, for each pair $(\boldsymbol{J}, \boldsymbol{b})$ mentioned in the introduction, there is a unique Riemannian metric $g$ conformal to $\boldsymbol{J}$ such that $g$ and $\boldsymbol{b}$ are, respectively, the Blaschke metric and the Pick differential of a convex projective structure. This gives the identification $\mathcal{P}(\Sigma) \cong \mathcal{C}(\Sigma)$ discussed in the introduction.

The entropy $\operatorname{Ent}\left(g_{\mathrm{H}}\right)$ of the Hilbert metric is an interesting and systematically studied quantity because it equals the topological entropy of an interesting dynamical system — the geodesic flow of a convex projective surface (see Cra09]).

As in the introduction, with an abuse of notation, we also let Ent $: \mathcal{P}(\Sigma) \rightarrow \mathbb{R}_{+}$ denote the function assigning to each convex projective structure the entropy of its Hilbert metric.

Proof of the "if" part of the theorem. Proposition 3.4 in BH13 implies that there is a universal constant $c>1$ such that the Hilbert metric $g_{\mathrm{H}}$ and the Blaschke metric $g_{\mathrm{B}}$ of any convex projective structure on $\Sigma$ satisfy

$$
c^{-1}\|v\|_{g_{\mathrm{B}}} \leq\|v\|_{g_{\mathrm{H}}} \leq c\|v\|_{g_{\mathrm{B}}}
$$

for any tangent vector $v$ of $\Sigma$. Here $\|v\|_{g_{\mathrm{B}}}$ and $\|v\|_{g_{\mathrm{H}}}$ denotes the norm of $v$ with respect to $g_{\mathrm{B}}$ and $g_{\mathrm{H}}$, respectively. As a consequence, we have

$$
c^{-1} \operatorname{Ent}\left(g_{\mathrm{B}}\right) \leq \operatorname{Ent}\left(g_{\mathrm{H}}\right) \leq c \operatorname{Ent}\left(g_{\mathrm{B}}\right) .
$$

Given a convex projective structure with Blaschke metric $g_{\mathrm{B}}$ and Pick differential $\boldsymbol{b}$, we consider the flat metric with conic singularity $|\boldsymbol{b}|^{\frac{2}{3}}$. By definition, if $\boldsymbol{b}=$ $b(z) \mathrm{d} z^{3}$ under a conformal local coordinate $z$, then $|\boldsymbol{b}|^{\frac{2}{3}}:=|b(z)|^{\frac{2}{3}}|\mathrm{~d} z|^{2}$.

It is a well-known fact that $g_{\mathrm{B}}$ has non-positive curvature (see e.g. Nie15, Cor. $6.2]$ ), or equivalently, there is a pointwise majorization

$$
g_{\mathrm{B}} \geq 2^{\frac{1}{3}}|\boldsymbol{b}|^{\frac{2}{3}}
$$

(the equivalence follows from Wang's equation (2.3)). Therefore we have

$$
\operatorname{Ent}\left(g_{\mathrm{B}}\right) \leq \operatorname{Ent}\left(2^{\frac{1}{3}}|\boldsymbol{b}|^{\frac{2}{3}}\right) \text {. }
$$

Let $\left(\boldsymbol{b}_{n}\right)$ be a sequence in $V=H^{0}\left((\Sigma, \boldsymbol{J}), K^{3}\right)$ tending to infinity. In view of (2.4) and (2.6), in order to prove the "if" part of the theorem, it is sufficient to show that $\operatorname{Ent}\left(2^{\frac{1}{3}}\left|\boldsymbol{b}_{n}\right|^{\frac{2}{3}}\right)$ tends to 0 .

To this end, we note that the function

$$
V \backslash\{0\} \rightarrow \mathbb{R}_{\geq 0}, \quad \boldsymbol{b} \mapsto \operatorname{Ent}\left(2^{\frac{1}{3}}|\boldsymbol{b}|^{\frac{2}{3}}\right)
$$

is continuous, because for $\boldsymbol{b}, \boldsymbol{b}^{\prime} \in V \backslash\{0\}$, the quasi-isometry constant $a$ in (2.1) between the lifts of $|\boldsymbol{b}|^{\frac{2}{3}}$ and $\left|\boldsymbol{b}^{\prime}\right|^{\frac{2}{3}}$ tends to 1 as $\boldsymbol{b}^{\prime}$ approaches $\boldsymbol{b}$. Therefore, if we fix a 
norm $\|\cdot\|$ on $V$ and let $S \subset V$ be the unit sphere, then $M:=\max _{\boldsymbol{b} \in S} \operatorname{Ent}\left(|\boldsymbol{b}|^{\frac{2}{3}}\right) \in \mathbb{R}_{+}$ exists. Thus,

$$
\begin{aligned}
\operatorname{Ent}\left(2^{\frac{1}{3}}\left|\boldsymbol{b}_{n}\right|^{\frac{2}{3}}\right)=\operatorname{Ent}\left(2^{\frac{1}{3}}\left\|\boldsymbol{b}_{n}\right\|^{\frac{2}{3}}\left|\frac{\boldsymbol{b}_{n}}{\left\|\boldsymbol{b}_{n}\right\|}\right|^{\frac{2}{3}}\right) & =2^{-\frac{1}{3}}\left\|\boldsymbol{b}_{n}\right\|^{-\frac{2}{3}} \operatorname{Ent}\left(\left|\frac{\boldsymbol{b}_{n}}{\left\|\boldsymbol{b}_{n}\right\|}\right|^{\frac{2}{3}}\right) \\
& \leq 2^{-\frac{1}{3}}\left\|\boldsymbol{b}_{n}\right\|^{-\frac{2}{3}} M .
\end{aligned}
$$

The last term tends to 0 because $\left\|\boldsymbol{b}_{n}\right\|$ tends to $+\infty$, as required.

In order to prove the "only if" part of the theorem without using the continuity of the Labourie-Loftin bijection, as mentioned in the introduction, we need the following lemma.

Lemma. Given a convex projective structure on $\Sigma$ with Blaschke metric $g_{\mathrm{B}}$ and Pick differential $\boldsymbol{b}$, the total area of $g_{\mathrm{B}}$ and that of the flat metric $2^{\frac{1}{3}}|\boldsymbol{b}|^{\frac{2}{3}}$ satisfy

$$
\operatorname{Area}\left(g_{\mathrm{B}}\right) \leq 2 \pi|\chi(\Sigma)|+\operatorname{Area}\left(2^{\frac{1}{3}}|\boldsymbol{b}|^{\frac{2}{3}}\right) \text {. }
$$

Proof. Wang's equation (2.3) satisfied by $g_{\mathrm{B}}$ and $\boldsymbol{b}$ can be written as

$$
1=-\kappa_{g_{\mathrm{B}}}+2\|\boldsymbol{b}\|_{g_{\mathrm{B}}}^{2} .
$$

Integrating both sides over $\Sigma$ with respect to the volume form of $g_{\mathrm{B}}$, then applying the Gauss-Bonnet formula to the first term on the right-hand side, we get

$$
\operatorname{Area}\left(g_{\mathrm{B}}\right)=2 \pi|\chi(\Sigma)|+\int_{\Sigma} 2\|\boldsymbol{b}\|_{g_{\mathrm{B}}}^{2} \mathrm{dvol}_{g_{\mathrm{B}}} .
$$

To prove the lemma, it is sufficient to show that the integrand $2\|\boldsymbol{b}\|_{g_{\mathrm{B}}}^{2} \mathrm{dvol}_{g_{\mathrm{B}}}$ in the last term above is pointwise majorized by the volume form dvol' of $2^{\frac{1}{3}}|\boldsymbol{b}|^{\frac{2}{3}}$, but this follows from the inequality (2.5): assuming $g_{\mathrm{B}}=h(z)|\mathrm{d} z|^{2}$ and $\boldsymbol{b}=b(z) \mathrm{d} z^{3}$ in a local coordinate $z=x+\boldsymbol{i} y$, we have

$$
2\|\boldsymbol{b}\|_{g_{\mathrm{B}}}^{2} \mathrm{dvol}_{g_{\mathrm{B}}}=\frac{2|b|^{2}}{h^{2}} \mathrm{~d} x \wedge \mathrm{d} y \leq \frac{2|b|^{2}}{\left(2^{\frac{1}{3}}|b|^{\frac{2}{3}}\right)^{2}} \mathrm{~d} x \wedge \mathrm{d} y=2^{\frac{1}{3}}|b|^{\frac{2}{3}} \mathrm{~d} x \wedge \mathrm{d} y=\mathrm{dvol}^{\prime} .
$$

Proof of the "only if" part of the theorem. Let $g_{\mathrm{B}}^{(n)}$ be the Blaschke metric of the convex projective structure corresponding to $\left(\boldsymbol{J}, \boldsymbol{b}_{n}\right)$ and assume that

$$
\lim _{n \rightarrow+\infty} \operatorname{Ent}\left(g_{\mathrm{B}}^{(n)}\right)=0 \text {. }
$$

We need to prove that the cubic differential $\boldsymbol{b}_{n}$ tends to infinity in $H^{0}\left((\Sigma, \boldsymbol{J}), K^{3}\right)$, or equivalently, the total area of the associated flat metric $\left|\boldsymbol{b}_{n}\right|^{\frac{2}{3}}$ tends to infinity. But this follows from Katok's inequality (2.2) and the above lemma, because they imply

$$
\frac{2 \pi|\chi(\Sigma)|}{\operatorname{Ent}\left(g_{\mathrm{B}}^{(n)}\right)^{2}} \leq \operatorname{Area}\left(g_{\mathrm{B}}^{(n)}\right) \leq 2 \pi|\chi(\Sigma)|+\operatorname{Area}\left(2^{\frac{1}{3}}\left|\boldsymbol{b}_{n}\right|^{\frac{2}{3}}\right) .
$$

This completes the proof of the theorem.

To conclude, we give some comments on the case where $\Sigma$ is a punctured surface of finite type, i.e. $\Sigma$ is obtained from a closed oriented surface by removing finitely many punctures. As a generalization of the Labourie-Loftin identification $\mathcal{P}(\Sigma) \cong$ $\mathcal{C}(\Sigma)$, Benoist and Hulin BH13] identified the space of convex projective structures with finite Hilbert volume with the space of those pairs $(\boldsymbol{J}, \boldsymbol{b})$ where $\boldsymbol{J}$ is a punctured 
Riemann surface structure and $\boldsymbol{b}$ is a holomorphic cubic differential with at most second order pole at each puncture. We gave in Nie15 a further generalization.

Perhaps, the above theorem holds in these more general settings as well. However, the above proof does not work. Indeed, at a pole of order $\leq 2$, the metric $|\boldsymbol{b}|^{\frac{2}{3}}$ is incomplete and its pullback to the universal cover is not quasi-isometric to the hyperbolic plane. Hence, whether or not $|\boldsymbol{b}|^{\frac{2}{3}}$ has finite entropy remains a problem.

\section{ACKNOWLEDGEMENT}

The authors would like to thank the referee for a helpful remark on the first draft of the paper.

\section{REFERENCES}

[BH13] Yves Benoist and Dominique Hulin, Cubic differentials and finite volume convex projective surfaces, Geom. Topol. 17 (2013), no. 1, 595-620, DOI 10.2140/gt.2013.17.595. MR.3039771

[Cra09] Mickaël Crampon, Entropies of strictly convex projective manifolds, J. Mod. Dyn. 3 (2009), no. 4, 511-547, DOI 10.3934/jmd.2009.3.511. MR2587084 (2011g:37079)

[Kat88] Anatole Katok, Four applications of conformal equivalence to geometry and dynamics, Ergodic Theory Dynam. Systems 8* (1988), no. Charles Conley Memorial Issue, 139-152, DOI 10.1017/S0143385700009391. MR967635 (89m:58165)

[Lab07] François Labourie, Flat projective structures on surfaces and cubic holomorphic differentials, Pure Appl. Math. Q. 3 (2007), no. 4, 1057-1099, DOI 10.4310/PAMQ.2007.v3.n4.a10. MR2402597(2009c:53046)

[Lof01] John C. Loftin, Affine spheres and convex $\mathbb{R P}^{n}$-manifolds, Amer. J. Math. 123 (2001), no. 2, 255-274. MR1828223 (2002c:53018)

[Lof07] John Loftin, Flat metrics, cubic differentials and limits of projective holonomies, Geom. Dedicata 128 (2007), 97-106, DOI 10.1007/s10711-007-9184-2. MR2350148 (2009c:53011)

[Nie] Xin Nie, On the Hilbert geometry of simplicial Tits sets, arXiv:0902.0885, to appear in Ann. Inst. Fourier (2015).

[Nie15] Xin Nie, Meromorphic cubic differentials and convex projective structures, ArXiv:1503.02608 (2015).

[Par12] Anne Parreau, Compactification d'espaces de représentations de groupes de type fini (French, with English summary), Math. Z. 272 (2012), no. 1-2, 51-86, DOI 10.1007/s00209-011-0921-8. MR.2968214

[Zha13] T. Zhang, The degeneration of convex RP^2 structures on surfaces, ArXiv:1312.2452 (2013).

School of Mathematics, KiAS, 85 Hoegiro, Dongdaemun-gu, Seoul 130-722, Republic OF KOREA.

E-mail address: nie.hsin@gmail.com 